\title{
RoboCup 2001 (F180) Team Description: RoboSix UPMC-CFA (France)
}

Francis Bras, Ryad Benosman, André Anglade, Seko Latidine, Olivier Martino, Aude Lagardere, Alain Boun, Alain Testa, and Gilles Cordurié

\author{
Université Pierre et Marie Curie \\ 4 place jussieu \\ 75252 Paris cedex 05, boite 164 \\ France \\ rbo@lis.jussieu.fr \\ http://www.robosix .com
}

\begin{abstract}
This paper describes the team RoboSix UPMC-CFA that participated to the German open 2001 and RoboCup 2001 in the F180league. Wel will present the mechanical and electrical design of the robots. We will then introduce the vision choices explaining the colometric and geometric calibrations stages. Finally we will explain the implemented behaviour and the path planning associated to it.
\end{abstract}

\section{Introduction}

RoboSix UPMC-CFA is a team composed of students from different sections of University Pierre and Marie Curie. The team has one electrical engineering student belonging to the engineering school IFITEP and four computer vision students belonging to the DESS IE. The team has three adivisors that have been involved in all Robocup's championships since Nagoya in 1997. The RoboCup UPMC-CFA 2001 team is a team that was created in september 1999. Our main motivation is to use Robocup as a test platform for new approaches in computer vision.

\section{Mechanical Design}

Our robots are designed to fit the new F180 size regulation. They are built out of aluminum frames that protect their inner parts. The robots have a differential drive with two active wheels that are not in the middle to allow a more complicated and unpredictable trajectories and a better twisting to shoot the ball. The maximum speed has been pushed to more than $2 \mathrm{~m} / \mathrm{s}$, by far more than needed by the vision. The robots carry a directional kicker, but due to a lack of time they were not used. The team has also an omnidirectional goalie, to avoid the problem of losing time while repositionning the player. 


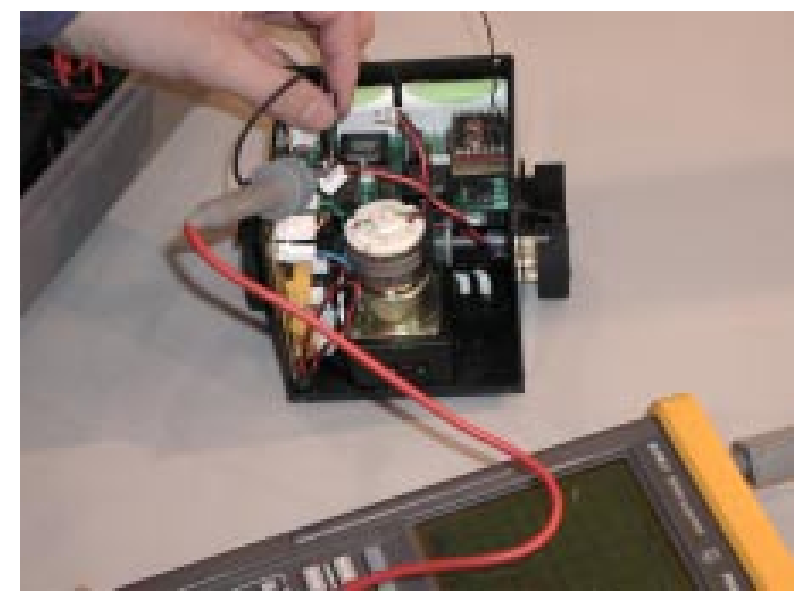

Fig. 1. Inside UPMC-CFA robot

\section{$3 \quad$ Electrical Design}

The microcontroller used is an Atmel 89S53 that has the advantage of beinging programmable in situ with $12 \mathrm{~kb}$ flash memory running at $24 \mathrm{Mhz}$. For radio communication we used the classical Radiometrix working in the band $433 \mathrm{Mhz}$ and $418 \mathrm{Mhz}$. The robots are powered by two Maxon motors with an integrated 19:1 gear, we used a dual $\mathrm{H}$ bridge motor driver L6202. The batteries were specially assembled to fit in the robots and are from GP Batteries our sponsor. Finally a Altera 7032 generates the pulse with modulation for the motors. All the described devices were mounted on a single custom card (see Fig 1) including the stabilized power supply.

\section{Computer Vision}

\subsection{Hardware Description}

We used a PCI-framegrabber and a progressive CCD camera. The chosen camera has the advantage of needing only one cable for data and power directly connected to the Pc. We capture RGB images of size $640 * 480$ at a rate of $30 \mathrm{fps}$. The system is running under Windows NT on a pentium $41.5 \mathrm{GHz}$.

\subsection{Color Calibration and Regions Labelling}

The output signal of the camera is RGB. Compared to the last year approach, we developped a new color calibration concept that was used by our both middle and small size teams. Classically a color is defined as a three component vector containing the values for the red, green and blue color planes. Generally the first 
calibration stage is manually made where colors are selected, for each color we compute a mean and a standard deviation. The image is then filtered, an image containing only the desired colors is generated. Last year a second detection based on HSV color space was applied only on the detected zones to reduce computation and increase robustness. This year the color calibration relies only on RGB. Instead of using a 3D cube to determine a color, we used 3D quadrics that are projected on three planes in the best oriented $3 \mathrm{D}$ coordinates system giving the best separation between close colors, this method will be published soon. The system is robust to change of lighting and has been succesfully tested.

\subsection{Recognizing Robots}

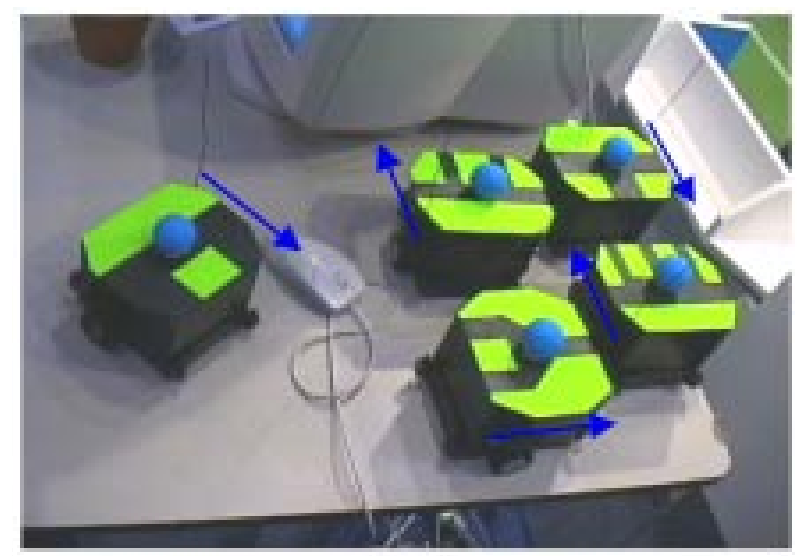

Fig. 2. Caraterizing the robots and their orientations

The coding of the robots is kept the same as last year and relies on the same bar code. Each robot has a number of thick and thin bars (see Fig 2). The bars are chosen so that we can have the information on the orientation and at the same time allowing to retreive the robot's position with a very high accuracy. The geometric calibration is widely explained in [1] and allows us to locate our robot on the table. The aim is to be able to retreive from the images' coordinates the position of the robots according to a coordinates system on the table taking into account the height of the observed object. It is based on projective geometry and more specifically colineations [2].

\subsection{Behaviour}

The behaviour of the robots is motivated by different flags corresponding to specific situations and robots' positions. The behaviour relies on the path finding 
procedure that uses a delaunay triangulation to find the best path taking into account the length of the best paths. The behaviour of the team relies on the same flags as last year. It analyzes the situation and assigns new position to be fullfilled. The path planning determines for each target which robot is the closest and generates the best path. Using such a system at the contrary of what has been used last year, no robot has an assigned position, even the goalie if wanted could switch position with a player if needed for an action. The behaviour of the robot varies according to situations for example if the team is winning or not.

\section{Conclusion}

We find robocup a very good tool for pedagogy and research. Unless we increase the size of the field, it is going to be harder and harder to have a cooperative game and score against defensive teams. This year, the team's level has highly improved, we see more and more passes. We are considering new techniques to allow more dynamic passes for next year..

\section{References}

1. Ryad Benosman and Jerome Douret: A simple and accurate camera calibration for the F180 RoboCup league RoboCup Symposium 2001, Seattle, USA

2. J.G Semple and G.T Kneebone Algebraic Projective Geometry, Oxford University Press 1952 